\title{
Modeling of laser-pulse induced water decomposition on two-dimensional materials by simulations based on time-dependent density functional theory
}

\author{
Yoshiyuki Miyamoto, ${ }^{1, *}$ Hong Zhang, ${ }^{2, \dagger}$ Xinlu Cheng, ${ }^{3}$ and Angel Rubio ${ }^{4,5}$ \\ ${ }^{1}$ Research Center for Computational Design of Advanced Functional Materials, National Institute of Advanced Industrial Science \\ and Technology, Central 2, 1-1-1 Umezono, Tsukuba, Ibaraki 305-8568, Japan \\ ${ }^{2}$ College of Physical Science and Technology, Sichuan University, Chengdu 610065, China \\ ${ }^{3}$ Key Laboratory of High Energy Density Physics and Technology of Ministry of Education, Sichuan University, Chengdu 610064, China \\ ${ }^{4}$ Max Planck Institute for the Structure and Dynamics of Matter and Center for Free-Electron Laser Science, \\ Luruper Chaussee 149, 22761 Hamburg, Germany \\ ${ }^{5}$ Nano-Bio Spectroscopy Group and ETSF, Universidad del País Vasco, CFM CSIC-UPV/EHU-MPC, 20018 San Sebastián, Spain
}

(Received 30 September 2016; revised manuscript received 4 September 2017; published 28 September 2017)

\begin{abstract}
We use time-dependent density functional theory to study laser-pulse induced decomposition of $\mathrm{H}_{2} \mathrm{O}$ molecules above the two-dimensional (2D) materials graphene, hexagonal boron nitride, and graphitic carbon nitride. We examine femtosecond-laser pulses with a full width at half maximum of 10 or $20 \mathrm{fs}$ for laser-field intensity and wavelengths of 800 or $400 \mathrm{~nm}$ by varying the intensity of the laser field from 5 to $9 \mathrm{~V} / \AA$, with the corresponding range of fluence per pulse up to $10.7 \mathrm{~J} / \mathrm{cm}^{2}$. For a $\mathrm{H}_{2} \mathrm{O}$ molecule above the graphitic sheets, the threshold for laser-field $\mathrm{H}_{2} \mathrm{O}$ decomposition is reduced by more than $20 \%$ compared with that of an isolated $\mathrm{H}_{2} \mathrm{O}$ molecule. We also show that hole doping enhances the water adsorption energy above graphene. The present results indicate that the graphitic materials should support laser-induced chemistry and that other 2D materials that can enhance laser-induced $\mathrm{H}_{2} \mathrm{O}$ decomposition should be investigated.
\end{abstract}

DOI: 10.1103/PhysRevB.96.115451

\section{INTRODUCTION}

Hydrogen fuel is expected to reduce carbon emissions, but the production yield needs to be improved to provide a stable, large-scale supply. Hydrogen production from an abundant $\mathrm{H}_{2} \mathrm{O}$ source is beneficial, and the discovery of UV-induced $\mathrm{H}_{2} \mathrm{O}$ decomposition on $\mathrm{TiO}_{2}$ [1] triggered the exploration of other materials that enhance photoinduced $\mathrm{H}_{2} \mathrm{O}$ decomposition as well as understanding of the mechanisms. Other transition-metal oxides, such as tantalite [2] and $\mathrm{RuO}_{2}$ [3-6], have been intensively studied. A metal-free class of photocatalytic materials [7], graphitic carbon nitride $\left(\mathrm{gC}_{3} \mathrm{~N}_{4}\right)$, was recently shown to decompose $\mathrm{H}_{2} \mathrm{O}$ with visible light. Although the yield of the $\mathrm{H}_{2} \mathrm{O}$ decomposition was much lower than for $\mathrm{TiO}_{2}$, the photoinduced decomposition was improved by combining $\mathrm{gC}_{3} \mathrm{~N}_{4}$ with carbon nanodots [8]. Graphitic materials are hydrophobic, but it was theoretically proposed that electric field doping could change their properties from hydrophobic to hydrophilic [9], which motivated us to investigate the effect of doping graphene sheets on $\mathrm{H}_{2} \mathrm{O}$ adsorption. Generally, photoinduced $\mathrm{H}_{2} \mathrm{O}$ decomposition is understood as a sequential process [10] in which photoexcited carriers in the catalytic material play an important role in $\mathrm{H}_{2} \mathrm{O}$ decomposition. This charge-transfer mechanism may also explain the electrochemical $\mathrm{H}_{2} \mathrm{O}$ decomposition on $\mathrm{gC}_{3} \mathrm{~N}_{4}$ sheets [11].

In this work, we examine a short, intense laser pulse as another method for $\mathrm{H}_{2} \mathrm{O}$ decomposition for higher $\mathrm{H}_{2}$ production per time under high photon flux, although it may increase energy consumption. Noble-metal clusters enhance the optical field for laser frequencies matching the plasmon resonance frequencies [12-15]. We propose that the enhancement also

\footnotetext{
*yoshi-miyamoto@aist.go.jp

†hongzhang@scu.edu.cn
}

occurs with graphitic materials consisting of earth-abundant elements. We should note that the enhancement was observed even without resonance with a particular plasmon mode in the graphitic materials. Our simulations indicate that graphitic layered materials, such as graphene, hexagonal boron nitride (hBN), and $\mathrm{gC}_{3} \mathrm{~N}_{4}$, enhance $\mathrm{H}_{2} \mathrm{O}$ decomposition compared with an isolated $\mathrm{H}_{2} \mathrm{O}$ molecule that represents the gas phase of water. The case of several $\mathrm{H}_{2} \mathrm{O}$ molecules and the presence of a monovacancy on the graphene sheets were also examined. The graphitic sheets are hydrophobic; however, our simulations show that hole doping can significantly increase graphene- $\mathrm{H}_{2} \mathrm{O}$ interaction. The simulation uses a femtosecond laser with a full width at half maximum (FWHM) of 10 or 20 fs for the laser-field intensity and wavelengths of 800 or $400 \mathrm{~nm}$ with the optical polarization vector normal to the plane of the layered materials. Taking many data by changing the parameters for FWHM or wavelength can produce a curve of the threshold as a function of these parameters, which is not within the current scope. A schematic of this polarization condition is shown in the right panel of Fig. 1(a).

By performing real-time propagation of the electron wave functions based on time-dependent density functional theory (TDDFT) [16] and molecular dynamics (MD) simulations in the presence of a femtosecond laser field of various intensities, we determined the threshold intensity for $\mathrm{H}_{2} \mathrm{O}$ decomposition. We found that the threshold is reduced by more than $20 \%$ when the $\mathrm{H}_{2} \mathrm{O}$ molecule is on a sheet of a graphitic material, such as graphene, $\mathrm{hBN}$, or $\mathrm{gC}_{3} \mathrm{~N}_{4}$. This conclusion is robust and independent of the choice of the exchange-correlation energy functional for density functional theory (DFT), that is, independent of the local-density approximation (LDA) or the generalized gradient approximation (GGA). However, it has been reported that the adsorption energy of $\mathrm{OH}$ and $\mathrm{H}$ species on two-dimensional (2D) materials depends on the choice of DFT functional [17]. This observation would be relevant to the 
(a)

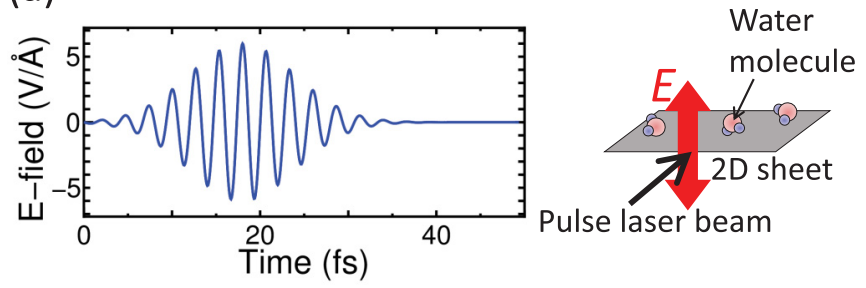

(b)

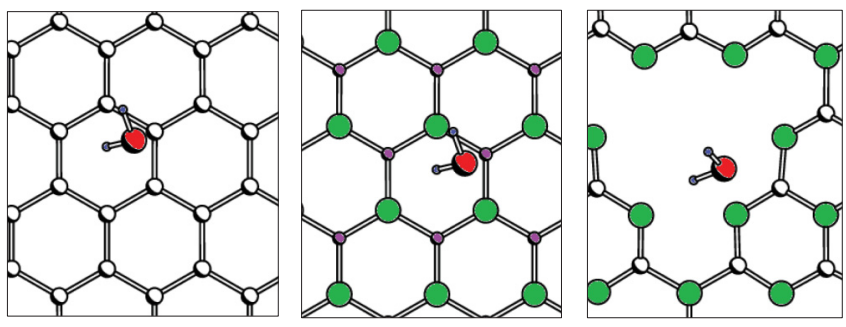

FIG. 1. (a) Left: Pulse shape of the E-field of the femtosecond laser with a wavelength of $800 \mathrm{~nm}$ and FWHM of $10 \mathrm{fs}$. In this display, the maximum E-field intensity is $6 \mathrm{~V} / \AA$. Right: Schematic explaining the polarization vector (red arrow) of the incident laser beam for the 2D sheet with several $\mathrm{H}_{2} \mathrm{O}$ molecules. (b) Starting geometry of an $\mathrm{H}_{2} \mathrm{O}$ molecule above the graphene sheet (left), hBN sheet (middle), and $\mathrm{gC}_{3} \mathrm{~N}_{4}$ sheet (right), determined by using the LDA functional. Open circles and hatched red circles denote carbon and oxygen atoms, respectively. Green (light gray) and violet (dark gray) circles denote nitrogen and boron atoms, respectively. The smallest hatched blue circles denote hydrogen atoms. All geometries are displayed from a viewing direction normal to the sheets.

present work if the study of laser-induced $\mathrm{H}_{2} \mathrm{O}$ decomposition were extended to the subsequent adsorption of the decomposed species on the $2 \mathrm{D}$ materials. However, in the current simulation, we did not observe any adsorption processes after applying the laser pulse. Simulation of the optical field near 2D materials upon laser irradiation shows an increase in the electric field (E-field) intensity by an amount consistent with the reduction factor of the threshold E-field intensity on the 2D sheets. Therefore, we conclude that optical E-field enhancement near 2D materials enhances the $\mathrm{H}_{2} \mathrm{O}$ decomposition.

In the following, we explain the computational methods in Sec. II and the results in Sec. III, for the cases of a single $\mathrm{H}_{2} \mathrm{O}$ molecule (Sec. III A) and higher $\mathrm{H}_{2} \mathrm{O}$ coverage (Sec. III B). Then, the present work is discussed in Sec. IV.

\section{COMPUTATIONAL METHODS}

$\mathrm{H}_{2} \mathrm{O}$ decomposition under a strong laser field was simulated by real-time TDDFT combined with an MD simulation within Ehrenfest dynamics [18]. The validity of this approach has been discussed in Refs. [19,20], in which the results without strong nonadiabatic coupling were expected to work properly. We solved the time-dependent Kohn-Sham equation

$$
i \hbar \frac{\partial \psi_{\mathrm{n}}^{K S}(\mathbf{r}, t)}{\partial t}=\left\{H^{K S}(\mathbf{r}, t)+V_{\mathrm{ext}}(\mathbf{r}, t)\right\} \psi_{\mathrm{n}}^{K S}(\mathbf{r}, t),
$$

where $\psi_{\mathrm{n}}(\mathrm{r}, t)$ is the time-dependent Kohn-Sham orbital and $H^{K S}(\mathrm{r}, t)$ is the Kohn-Sham Hamiltonian, which is a functional of the time-dependent charge density $\rho(\mathbf{r}, t)$ consisting of the sum of the norm of occupied Kohn-Sham orbitals. $V_{\text {ext }}(\mathbf{r}, t)$ incorporates the applied optical electric field [21] that interacts with both electrons and ions. In the present work, the external field is treated in the dipole approximation by using the Coulomb gauge. The LDA with the Perdew-Zunger functional [22] and the GGA with the Perdew-Burke-Ernzerhof (PBE) functional [23] were used as the exchange-correlation energy functionals. A plane-wave basis set with a cutoff energy of 71 Ry was used to express the Kohn-Sham orbitals. The time evolution was computed within the fourth-order split-operator scheme $[24,25]$ with a 0.03 -a.u. $\left(7.26 \times 10^{-4} \mathrm{fs}\right)$ time step. The total energy and forces were computed with the momentum-space formalism [26]. This scheme provides a total energy precision of $3 \times 10^{-5} \mathrm{eV}$ per atom and a force precision of $0.05 \mathrm{eV} / \AA$. The interaction between ions and valence electrons was expressed by using the norm-conserving pseudopotentials [27] with separable forms [28]. To compute an $\mathrm{H}_{2} \mathrm{O}$ molecule above the graphene or hBN sheets, a $3 \times 3$ unit cell was used, whereas for $\mathrm{H}_{2} \mathrm{O}$ above the $\mathrm{gC}_{3} \mathrm{~N}_{4}$ sheet, a primitive cell containing six carbon and eight nitrogen atoms was used. In all cases, we used four irreducible $k$ points for the momentum-space integration, corresponding to 21 irreducible $k$ points in the Brillouin zone of the $1 \times 1$ unit cell including the $\Gamma, M$, and $K$ points. A $12-\AA$ vacuum region was set in the sheet normal direction. The calculations were performed with the FPSEID code [29], and numerical stability was checked with the criterion of the energy-conservation rule with the presence of a dynamical external field [30], the polarity of which was smoothly reversed in the middle of the vacuum region to satisfy the periodic boundary condition. A larger $5 \times 5$ unit cell, as used in Ref. [17], was tested for $\mathrm{H}_{2} \mathrm{O}$ decomposition above a graphene sheet with a vacuum thickness of $12 \AA$ and cutoff energy of 71 Ry by using the $\Gamma$ point. This larger cell gave a threshold laser field intensity for the decomposition that was $10 \%$ less than that using the $3 \times 3$ cell. Therefore, the enhancement factor obtained using the $3 \times 3$ cell should be a lower-bounded quantity.

\section{RESULTS}

The left panel of Fig. 1(a) shows the E-field of the laser pulse with a wavelength of $800 \mathrm{~nm}$ and FWHM of $10 \mathrm{fs}$ applied to an $\mathrm{H}_{2} \mathrm{O}$ molecule above the 2D materials, and the right panel shows a schematic of the experimental setup of the polarization vector of the incident pulse laser, which is perpendicular to a $2 \mathrm{D}$ sheet. Here, we have performed a simulation of $\mathrm{H}_{2} \mathrm{O}$ molecules with up to five per $3 \times 3$ cell of graphene to give inter $-\mathrm{H}_{2} \mathrm{O}$ distances closer to those in liquid water in order to examine the influence of inter- $\mathrm{H}_{2} \mathrm{O}$ interaction on the decomposition. We changed the maximum E-field intensity at intervals of $0.5 \mathrm{~V} / \AA$ and monitored the $\mathrm{H}_{2} \mathrm{O}$ decomposition. Figure 1(b) shows our initial configuration of an $\mathrm{H}_{2} \mathrm{O}$ molecule on graphene, $\mathrm{hBN}$, and $\mathrm{gC}_{3} \mathrm{~N}_{4}$ sheets. The ionic velocities were set to zero at the start of the simulation.

\section{A. One $\mathrm{H}_{2} \mathrm{O}$ molecule, isolated and above $2 \mathrm{D}$ sheets}

We confirmed that decomposition of an isolated $\mathrm{H}_{2} \mathrm{O}$ molecule occurred with a maximum E-field intensity of $9 \mathrm{~V} / \AA$ 
using the LDA and the GGA with the PBE functional (see Supplemental Material [31]). A field intensity of up to $9 \mathrm{~V} / \AA$ may sound high; it corresponds to $5.37 \mathrm{~J} / \mathrm{cm}^{2}$ with FWHM = $10 \mathrm{fs}$ for the laser field, which can be achieved with a laser fluence per shot of $0.337 \mu \mathrm{J}$ with a beam diameter of $1 \mu \mathrm{m}$. It is noted that such high intensity should damage almost all materials; however, as will be shown in this work, it is suggested that controlling the polarization vector may avoid the damage.

The threshold intensity of the laser E-field for $\mathrm{H}_{2} \mathrm{O}$ decomposition was reduced considerably when an $\mathrm{H}_{2} \mathrm{O}$ molecule was located above perfect two-dimensional materials. Figures 2(a) to 2(c) show the time evolution of the $\mathrm{O}-\mathrm{H}$ bond lengths of an $\mathrm{H}_{2} \mathrm{O}$ molecule above graphene, $\mathrm{hBN}$, and $\mathrm{gC}_{3} \mathrm{~N}_{4}$ computed by LDA. Similar results and dynamics above graphene were obtained by GGA with the PBE functional (see Supplemental Material [31]). The thresholds for the decomposition were calculated as 6.0,6.0, and 7.0 V/ $\AA$ for graphene, hBN, and $\mathrm{gC}_{3} \mathrm{~N}_{4}$, respectively. The trajectory of $\mathrm{H}_{2} \mathrm{O}$ decomposition above a graphene sheet is shown in Figs. 2(d) to 2(h) at time intervals of $10 \mathrm{fs}$, during which no damage to the graphene sheet occurred. The dynamics of the $\mathrm{H}_{2} \mathrm{O}$ decomposition were similar to the case of an isolated $\mathrm{H}_{2} \mathrm{O}$ molecule: the decomposition was mediated by the breaking of one of the two $\mathrm{O}-\mathrm{H}$ bonds. The unbroken $\mathrm{O}-\mathrm{H}$ bond was directed toward the graphene sheet [Figs. 2(d) to $2(\mathrm{~g})$ ], and at 2.8-2.9 $\AA$ above the sheet, its $\mathrm{O}-\mathrm{H}$ axis turned, and then it moved away from the graphene sheet [Fig. 2(h)]. This trend in the dynamics was also observed for $\mathrm{hBN}$ and $\mathrm{gC}_{3} \mathrm{~N}_{4}$. When the laser intensity was increased, two $\mathrm{H}$ atoms left the $\mathrm{O}$ atom, and the graphitic $2 \mathrm{D}$ sheets were not damaged. From the present
TABLE I. Threshold intensity of the laser field for $\mathrm{H}_{2} \mathrm{O}$ decomposition with a wavelength of $800 \mathrm{~nm}$ and a FWHM of 10 or $20 \mathrm{fs}$.

\begin{tabular}{|c|c|c|c|c|}
\hline \multirow[b]{2}{*}{ FWHM (fs) } & \multicolumn{4}{|c|}{ Threshold intensity (V/Å) } \\
\hline & Isolated & On graphene & On hBN & On $\mathrm{gC}_{3} \mathrm{~N}_{4}$ \\
\hline 10 & 9.0 & 6.0 & 6.0 & 7.0 \\
\hline 20 & 8.0 & 5.0 & 5.0 & 5.5 \\
\hline
\end{tabular}

conclusion, one may expect that irradiating the laser pulse for the decomposition will not destroy the $2 \mathrm{D}$ sheet and thus it is repeatable. The practical situation will be discussed later.

We interpret the decomposition dynamics as driven by the excitation of electrons involved in the $\mathrm{O}-\mathrm{H}$ bonds of the $\mathrm{H}_{2} \mathrm{O}$ molecule. The excitation promotes electrons to transient high levels, which may have an $\mathrm{O}-\mathrm{H}$ antibonding character, allowing an $\mathrm{H}$ atom to leave. The leaving $\mathrm{H}$ atom was neutral according to the spatial integration of the electron density, which is consistent with the mechanism of the $\mathrm{O}-\mathrm{H}$ bond breaking being antibonding excitation rather than a Coulomb explosion.

We investigated how the threshold intensity depends on pulse width (FWHM) by performing the simulation with a FWHM of $20 \mathrm{fs}$ and the same wavelength $(800 \mathrm{~nm})$. Table I summarizes the results, showing a further reduction of the threshold with a wider FWHM. However, the reduction for a wider FWHM does not mean a reduction in laser fluence. The laser fluence is proportional to the laser power multiplied by the FWHM, and the laser power is proportional to the square of the E-field. Therefore, the threshold values listed in Table I (a)

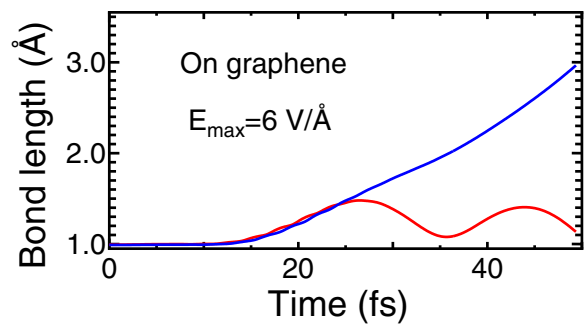

(d)

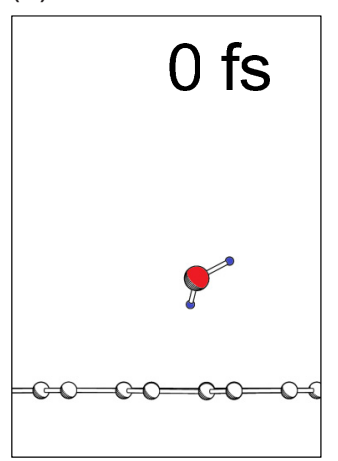

(b)

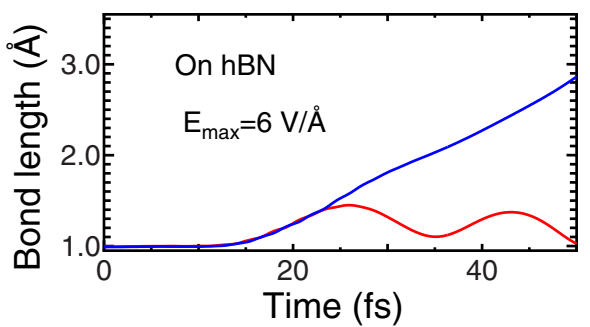

(f)

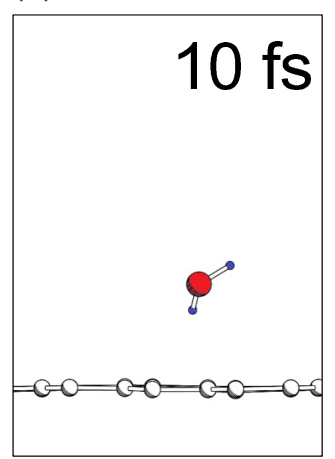

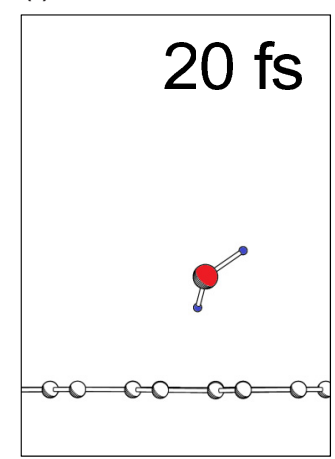

( (c)

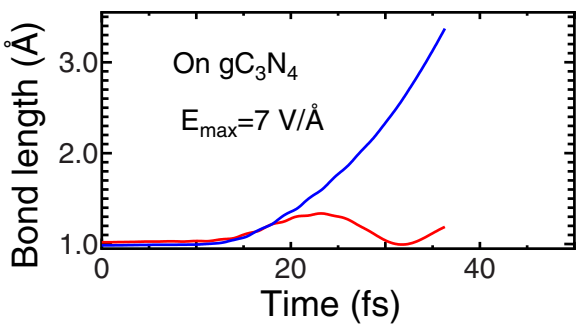

(h)

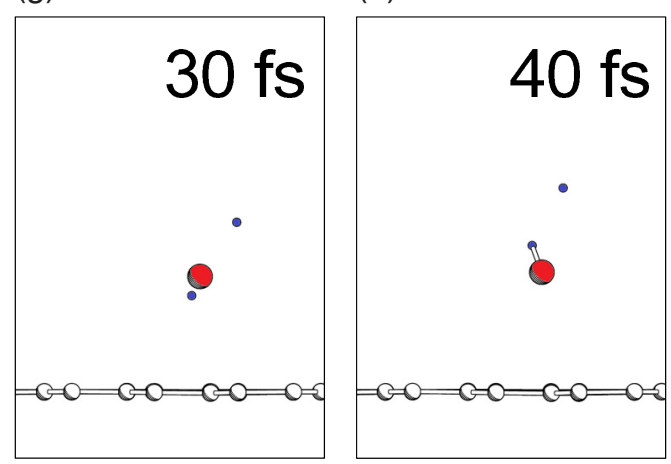

FIG. 2. Time evolution of the $\mathrm{O}-\mathrm{H}$ bond lengths of $\mathrm{H}_{2} \mathrm{O}$ molecules above (a) graphene, (b) $\mathrm{hBN}$, and (c) $\mathrm{gC}_{3} \mathrm{~N}_{4}$ sheets under the pulse laser with a FWHM of $10 \mathrm{fs}$. The corresponding threshold intensities of the laser E-field are also shown. One of the O-H bonds (blue curve) was broken in all cases. (d) to (h) Snapshots of $\mathrm{H}_{2} \mathrm{O}$ decomposition dynamics above a graphene sheet at 0, 10, 20, 30, and 40 fs, respectively. All geometries are displayed from a viewing direction parallel to the sheets. 
mean that the fluence necessary for $\mathrm{H}_{2} \mathrm{O}$ decomposition is smaller for a narrower FWHM. Thus, we recommend a shorter-pulse laser for $\mathrm{H}_{2} \mathrm{O}$ decomposition with lower energy consumption.

The threshold intensity for $\mathrm{H}_{2} \mathrm{O}$ decomposition above the graphene sheet was also examined by using the PBE functional form of the GGA. A simulation with a FWHM of $10 \mathrm{fs}$ and a wavelength of $800 \mathrm{~nm}$ gave a threshold intensity of $6.5 \mathrm{~V} / \AA$, which is close to the value obtained by LDA. Therefore, the current conclusion is robust with respect to the choice of the DFT functional. The details for real-time TDDFT-MD results using the PBE functional are presented in the Supplemental Material [31] (Figs. S1 and S2).

To identify the cause of the lower laser intensity threshold of $\mathrm{H}_{2} \mathrm{O}$ decomposition above graphitic materials, the enhancement of the optical field was examined [32,33]. We compared data for the applied and total (applied plus induced) optical field with a FWHM of $10 \mathrm{fs}$ and wavelength of $800 \mathrm{~nm}$ near graphene, $\mathrm{hBN}$, and $\mathrm{gC}_{3} \mathrm{~N}_{4}$ sheets. The total E-field was averaged in the sheet-parallel direction $3.34 \AA$ above and below the sheet, and the time variation of the E-field was plotted. There was a static E-field near the sheet from the gradient of the self-consistent potential for the electrons. By taking only the time variation of the total E-field, we excluded the value of the static E-field to highlight the amplitude modulation of the dynamical E-field. The results, obtained by LDA, are shown in Fig. 3. The applied field was $6 \mathrm{~V} / \AA$, and the maximum intensity of the total E-field was $8 \mathrm{~V} / \AA$ in all cases; thus, the enhancement factor was 1.33 . The threshold intensity of the $\mathrm{H}_{2} \mathrm{O}$ decomposition was reduced from 9 to $6 \mathrm{~V} / \AA$ when an $\mathrm{H}_{2} \mathrm{O}$ molecule was situated above the graphene and hBN sheets and was reduced to $7 \mathrm{~V} / \AA$ above the $\mathrm{gC}_{3} \mathrm{~N}_{4}$ sheet. This threshold reduction can be interpreted as E-field enhancement by a factor of 1.5 above graphene and $\mathrm{hBN}$ and by a factor of 1.28 above $\mathrm{gC}_{3} \mathrm{~N}_{4}$. These values are close to the enhancement factor of 1.33 obtained from the results in Fig. 3, and the subtle difference may originate from nonuniformity in the induced optical E-field due to the presence of an $\mathrm{H}_{2} \mathrm{O}$ molecule and the porous nature of the $\mathrm{gC}_{3} \mathrm{~N}_{4}$ sheet.

It should be noted that the optical field enhancement is usually driven by a resonance between the optical frequency and the excitation energy of the system, as was studied in gold nanoparticles [12-15], in semiconductor carbon nanotubes [32], and in graphene nanoribbons [33]. On the other hand, the current case shows that this enhancement cannot simply be explained by resonance. Instead, it should be understood by the intrinsic polarizability perpendicular to these $2 \mathrm{D}$ sheets.

Because the graphitic sheets studied here are hydrophobic, we sought a way to increase the interaction between $\mathrm{H}_{2} \mathrm{O}$ molecules and the graphitic sheet. Jiang et al. [9] theoretically demonstrated that electric field doping of graphene reduces the reaction barrier of $\mathrm{H}_{2} \mathrm{O}$ decomposition into $\mathrm{H}$ and $\mathrm{OH}$ species. Based on this report, we investigated whether the $\mathrm{H}_{2} \mathrm{O}$-graphene adsorption energy can be increased by electric field doping, which was mimicked by the injection of excess electrons or holes. Without doping, the adsorption energy of the $\mathrm{H}_{2} \mathrm{O}$ molecule to the graphene sheet was calculated as 88 and 13 meV using the LDA and GGA (PBE), respectively. The LDA (PBE) calculation showed a substantial increase in the adsorption energy to $0.20 \mathrm{eV}(0.50 \mathrm{eV})$ with an injection of one hole per $3 \times 3$ cell of the graphene sheet followed by a change in $\mathrm{H}_{2} \mathrm{O}$ orientation directing the $\mathrm{O}$ atom closer to the graphene sheet. This reorientation is consistent with the polar nature of $\mathrm{H}_{2} \mathrm{O}$ molecules. The assumed doping level corresponded to a downward shift of the Fermi level by $1.4 \mathrm{eV}$. (Meanwhile, injection of one electron per $3 \times 3$ cell of graphene gave an adsorption energy per $\mathrm{H}_{2} \mathrm{O}$ molecule similar to that in the case of nondoping using LDA and PBE functionals). The larger adsorption energy with hole doping suggests that electrostatic Coulomb forces are the dominant binding mechanism instead of van der Waals forces (relevant for the undoped case). Since a graphene sheet has a nonpolar
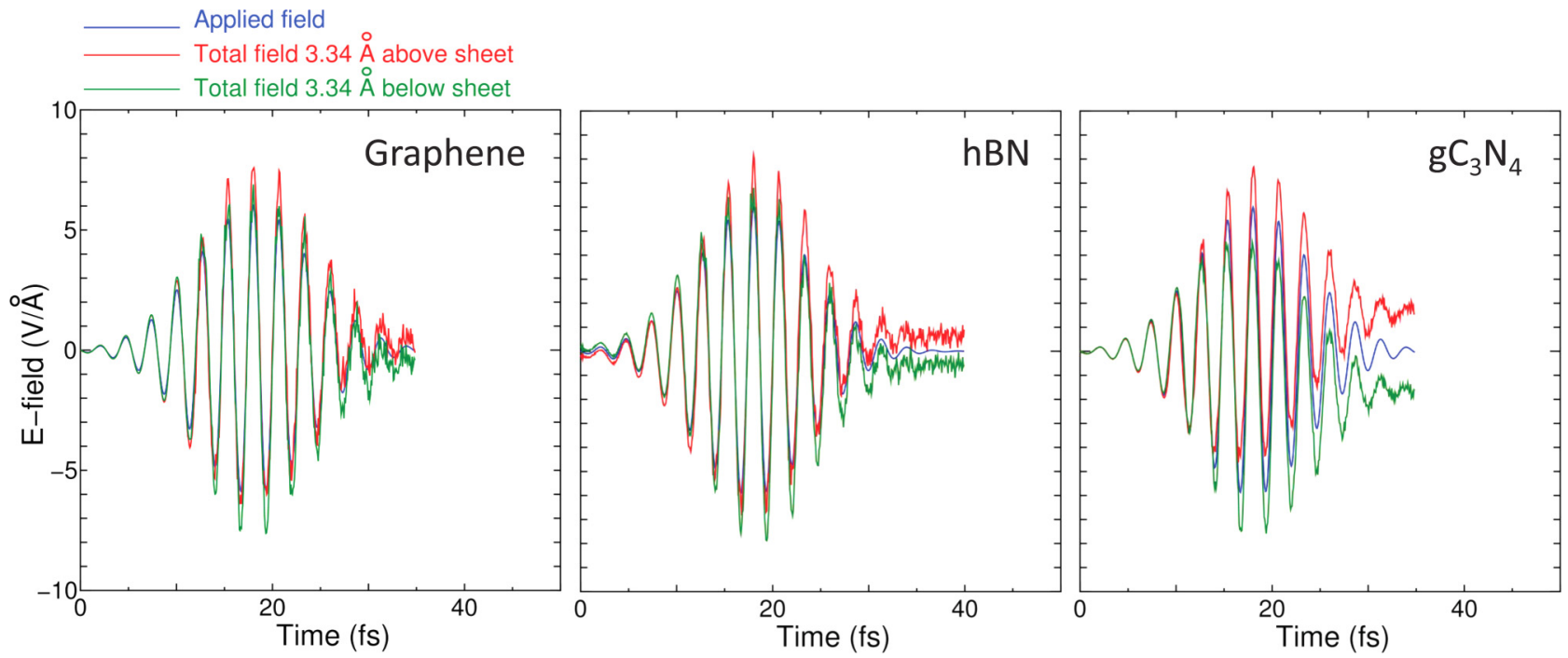

FIG. 3. Comparison of results for applied and total (applied plus induced) optical field $3.34 \AA$ above and below the sheets of graphene(left), $\mathrm{hBN}$ (middle), and $\mathrm{gC}_{3} \mathrm{~N}_{4}$ (right) for a femtosecond laser pulse with FWHM of $10 \mathrm{fs}$ and a wavelength of $800 \mathrm{~nm}$. The maximum field intensity of the applied field was set to $6 \mathrm{~V} / \AA$. Thick solid blue lines are the time evolution of the applied field, and the thin solid red and thin green lines are the time evolution of the total fields above and below the sheets, respectively. 
(a)

(b)

(c)

(d)

(e)

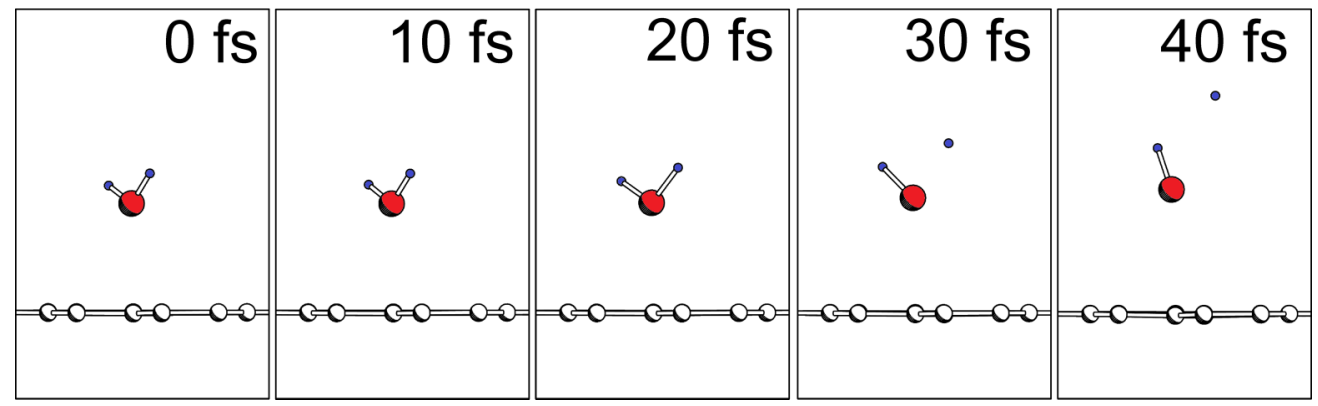

FIG. 4. Snapshots of water decomposition above a hole-doped graphene sheet at (a) 0, (b) 10, (c) 20, (d) 30, and (e) 40 fs. All geometries are displayed from a viewing direction parallel to the sheets. The orientation of the $\mathrm{H}_{2} \mathrm{O}$ molecule at $t=0$ fs is different from that shown in Fig. 2(d).

nature, we expect the $\mathrm{H}_{2} \mathrm{O}$ adsorption energy to weakly depend on the adsorption site. (This would not be the case for the polar $2 \mathrm{D}$ systems $\mathrm{hBN}$ and $\mathrm{gC}_{3} \mathrm{~N}_{4}$ ). If an increase in $\mathrm{H}_{2} \mathrm{O}$-graphene interaction is the case, it is expected to be beneficial for increasing the number of $\mathrm{H}_{2} \mathrm{O}$ molecules per area on the graphene sheet. The efficiency of $\mathrm{H}_{2} \mathrm{O}$ decomposition with higher $\mathrm{H}_{2} \mathrm{O}$ coverage is discussed in Sec. III B.

We confirmed that the laser decomposition of the $\mathrm{H}_{2} \mathrm{O}$ molecule also occurs under the hole-doping conditions using the LDA. Figures 4(a)-4(e) show the sequence of the decomposition dynamics with an E-field intensity of $7 \mathrm{~V} / \AA$, which is still considerably below the threshold of the decomposition of an isolated $\mathrm{H}_{2} \mathrm{O}$ molecule of $9 \mathrm{~V} / \AA$. The large increase in the adsorption energy of the $\mathrm{H}_{2} \mathrm{O}$ molecules indicates that the combination of electrical doping on graphene and the femtosecond laser should cause efficient water decomposition.

So far, we have tested the water decomposition by performing single-trajectory TDDFT-MD simulations, while a statistical ensemble of multitrajectory MD is necessary for high precision. We performed multitrajectory MD with higher $\mathrm{H}_{2} \mathrm{O}$ coverage, and the results are presented in the next section.

\section{B. With higher $\mathrm{H}_{2} \mathrm{O}$ coverage and with the presence of a defect on the graphene sheet}

At present, we have shown laser-induced decomposition of a single water molecule above graphitic sheets, but the environment of a water solution is of general interest. Compromising with computational cost, we have employed the LDA functional and used a model of five $\mathrm{H}_{2} \mathrm{O}$ molecules per $3 \times 3$ graphene cell. The top panels of Fig. 5 show three different atomic configurations of five $\mathrm{H}_{2} \mathrm{O}$ molecules above the $3 \times 3$ graphene cell, which were obtained by relaxing the atomic configurations starting with those generated by randomized numbers for expressing the location and orientation of $\mathrm{H}_{2} \mathrm{O}$ molecules. We compromised and restricted ourselves to the three different initial conditions to mimic the stochastic effect due to the limitation of the computational cost. The cohesive energy per one $\mathrm{H}_{2} \mathrm{O}$ molecule was calculated with the following equation:

$$
E(\text { graph })+5 E\left(\mathrm{H}_{2} \mathrm{O}\right)-E\left(\text { graph with } 5 \mathrm{H}_{2} \mathrm{O}\right),
$$

where $E$ (graph) and $E\left(\mathrm{H}_{2} \mathrm{O}\right)$ are the total energy of a pristine $3 \times 3$ graphene cell and that of an isolated $\mathrm{H}_{2} \mathrm{O}$ molecule, respectively. $E$ (graph with $5 \mathrm{H}_{2} \mathrm{O}$ ) is the total energy of five $\mathrm{H}_{2} \mathrm{O}$ molecules above a $3 \times 3$ graphene cell. The calculated values using LDA for the three atomic configurations were in the range $0.656 \pm 0.0033 \mathrm{eV}$, which is dominated by $\mathrm{H}_{2} \mathrm{O}-\mathrm{H}_{2} \mathrm{O}$ attraction in addition to $\mathrm{H}_{2} \mathrm{O}$-graphene interaction. By counting hydrogen bond energy as $0.25 \mathrm{eV}$ within the LDA level [34], the computed value is understandable via the formation of a two-dimensional network of hydrogen bonds. ( We know that the PBE functional is known to present a better value for the hydrogen bonding of $\mathrm{H}_{2} \mathrm{O}$ molecules [34], while the precision of the $\mathrm{H}_{2} \mathrm{O}$-graphene interaction obtained with LDA and PBE functionals is not guaranteed). (a)

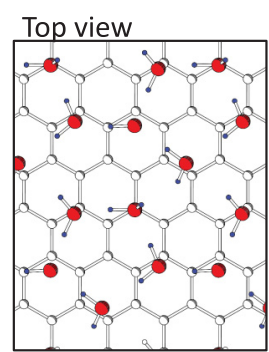

Side view

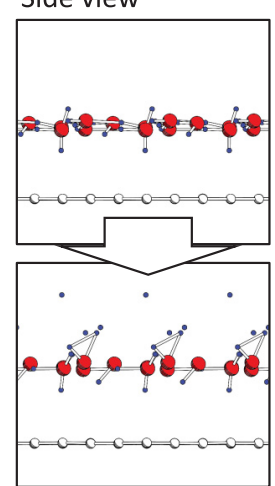

(b)

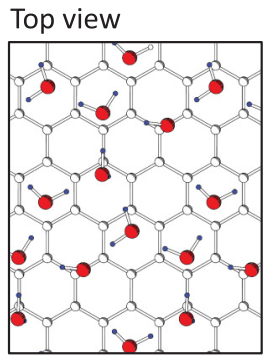

Side view

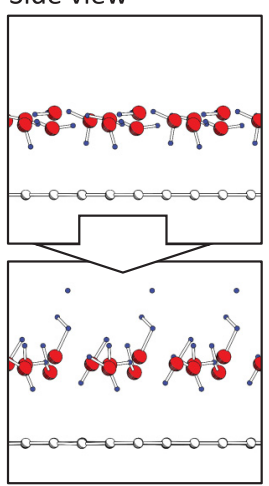

(c)

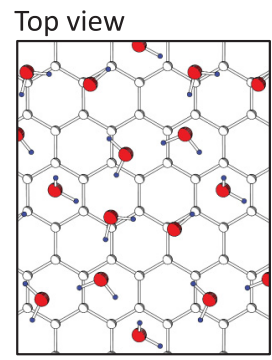

Side view

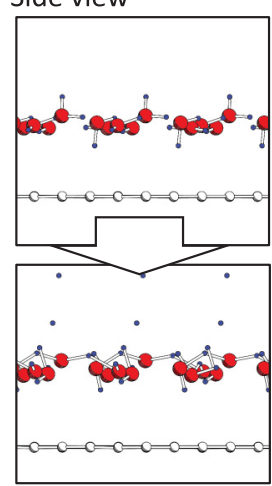

FIG. 5. (a)-(c) Cases of three different atomic configurations of five $\mathrm{H}_{2} \mathrm{O}$ molecules above a $3 \times 3$ graphene cell. The top panels show the top views of the three configurations, while the middle panels show side views. The bottom panels show the TDDFT-MD simulation 39 fs later under a pulse laser with an E-field of $6.5 \mathrm{~V} / \AA$. 


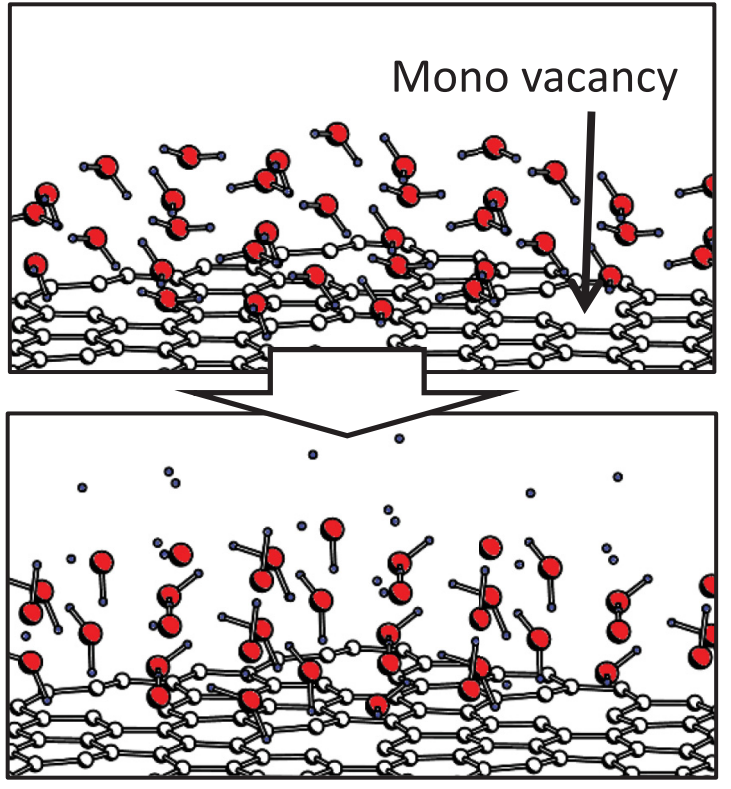

FIG. 6. Top: Five $\mathrm{H}_{2} \mathrm{O}$ molecules above a graphene sheet with monovacancy per $3 \times 3$ period. Bottom: Forty femtoseconds after the TDDFT-MD simulation with the presence of a pulse laser with $\mathrm{FWHM}=10 \mathrm{fs}$, a wavelength of $800 \mathrm{~nm}$, and an E-field of $6.5 \mathrm{~V} / \AA$.

For these three atomic configurations, the dynamics under a pulse laser with a FWHM $=10$ fs for the laser field and a wavelength of $800 \mathrm{~nm}$ was simulated as shown in the bottom panels of Fig. 5, which show the case with an optical E-field of $6.5 \mathrm{~V} / \AA$. (When the optical field was set to $6 \mathrm{~V} / \AA$, no $\mathrm{H}_{2} \mathrm{O}$ molecules were decomposed). This threshold intensity of $6.5 \mathrm{~V} / \AA$ is close to the value for an $\mathrm{H}_{2} \mathrm{O}$ molecule above a $3 \times 3$ graphene cell, $6 \mathrm{~V} / \AA$. We therefore concluded that the laser-pulse induced $\mathrm{H}_{2} \mathrm{O}$ decomposition is weakly dependent on the inter-water-molecule hydrogen bonding. Note that at the threshold intensity, not all five $\mathrm{H}_{2} \mathrm{O}$ molecules were decomposed.

In order to obtain a high decomposition yield, $\mathrm{H}_{2} \mathrm{O}$ condensation above graphitic sheets will be beneficial but requires a laser intensity beyond the threshold. Under the condition of the water density in the current simulation, a proton generated by laser decomposition of one $\mathrm{H}_{2} \mathrm{O}$ molecule did not contribute to decomposing the other $\mathrm{H}_{2} \mathrm{O}$ molecules. However, if an interface between the water liquid and the 2D sheet were considered, the proton dynamics would play an important role in $\mathrm{H}_{2} \mathrm{O}$ decomposition even after the decay of the laser field.

So far, in all the cases in the present work, the graphitic 2D sheet has shown no atomic-scale damage throughout the TDDFT-MD simulation despite the presence of a strong laser field, which may be due to the absence of defects in the present models. A natural question here is whether damage occurs when graphene has an atomic defect. To examine the stability of graphene, we introduced a single vacancy per $3 \times 3$ cell of the graphene sheet with a loading of five $\mathrm{H}_{2} \mathrm{O}$ molecules. Again, a pulse laser with FWHM $=10$ fs and a wavelength of $800 \mathrm{~nm}$ was applied with a laser intensity of $6.5 \mathrm{~V} / \AA$. Figure 6 shows the computed results $40 \mathrm{fs}$ after the TDDFT-MD simulation. As shown in Fig. 6, the atomic geometry of graphene sheets was unchanged even in the presence of a monovacancy, which was checked up to 80 fs. So direct destruction of the graphene sheet is very unlikely, which may be due to the current condition of laser field polarization perpendicular to the sheet. Meanwhile, the pulse laser generates $\mathrm{OH}$ radicals that depart from the graphene within the simulated time scale. However, for a long time, the $\mathrm{OH}$ radical may return to the graphene through migration. If that is the case, the $\mathrm{OH}$ radical strongly interacts with the monovacancy, as was studied in cases of oxidation of defective carbon nanotubes [35].

\section{DISCUSSION AND CONCLUSIONS}

We have shown pulse-laser induced $\mathrm{H}_{2} \mathrm{O}$ decomposition above graphitic $2 \mathrm{D}$ sheets. The $2 \mathrm{D}$ sheet did not suppress the decomposition, and even reduction of the laser intensity threshold for the decomposition was indicated by the present TDDFT-MD simulations. We further note that changing the wavelength from 800 to $400 \mathrm{~nm}$ reduced the threshold intensity. For example, with the LDA functional in the real-time TDDFT-MD simulation, the threshold of $\mathrm{H}_{2} \mathrm{O}$ decomposition above the graphene sheet was reduced to $E_{\max }=5 \mathrm{~V} / \AA$ with a wavelength of $400 \mathrm{~nm}$ and a FWHM of $10 \mathrm{fs}$ from $E_{\max }=6 \mathrm{~V} / \AA$ with a wavelength of $800 \mathrm{~nm}$ and the same FWHM. The reduction of the threshold intensity of the laser field could be due to the increase in photon energy at a shorter wavelength.

It is practically important to consider the yield of $\mathrm{H}_{2} \mathrm{O}$ decomposition, even when the intensity of the optical E-field is below the threshold due to thermal dynamics. For example, the $\mathrm{H}_{2} \mathrm{O}$ dynamics above graphene with an E-field intensity of $5.5 \mathrm{~V} / \AA$, below the threshold, shows an $\mathrm{O}-\mathrm{H}$ oscillation with an amplitude of $0.4 \AA$. This amplitude is large and should be comparable to thermodynamics at temperatures around $1000 \mathrm{~K}$; however, quantifying the yield of $\mathrm{H}_{2} \mathrm{O}$ decomposition requires a longer time and larger size simulation, which is left to future work. With this condition, the kinetic energy of hydrogen atoms reaches $0.75 \mathrm{eV}$, which should be in the range in which the proton ions can be treated using Newton's dynamics. (The current Ehrenfest MD approach in such a long-time simulation will be validated by using the method in Ref. [20]).

The results of this study suggest that using $2 \mathrm{D}$ materials can reduce the laser power for $\mathrm{H}_{2} \mathrm{O}$ decomposition, which helps increase the laser-beam cross section for the decomposition. The results also suggest that the nature of the graphitic sheets can be changed from hydrophobic to hydrophilic by electrical doping of holes. Although the factor of enhancement of $20 \%$ may not be significant, other materials, like the outer region of carbon nanotubes (CNTs), may be effective for higher field enhancement because of the field concentration due to the curvature of the CNT walls. To attract $\mathrm{H}_{2} \mathrm{O}$ molecules to such a curved region, the use of surfactants may be necessary [36]. Expanding the current research to other 2D materials, such as $\mathrm{MoS}_{2}$, is also important. The currently obtained threshold of field intensity for decomposition may sound high, but it is expected that one can reduce the value with a higher-magnitude FWHM, which is left to future work because of the computational costs. 


\section{ACKNOWLEDGMENTS}

Calculations were performed with the Parallel Computing System, AIST; the High-Performance Computing System of the Cybermedia Center, Osaka University; and the Cyberscience Center, Tohoku University. Y.M. thanks M. Kakehata for discussions about the experimental accessibility of current femtosecond lasers and acknowledges funding from JSPS KAKENHI Grants No. JP16H00925, No. JP16H04103, and No. JP16K05049. Y.M. also acknowledges support from the
Research Organization of Information Science and Technology (RIST), Tokyo. H.Z. and X.C. acknowledge financial support from the National Key R\&D Program of China 2017YFA0303603 and the National Natural Science Foundation of China (Grants No. 11474207 and No. 11374217). A.R. acknowledges financial support from the JSPS Fellowship program and from the European Research Council (QSpecNewMat ERC-2015-AdG-694097) and Grupos Consolidados (IT578-13).
[1] T. Inoue, A. Fujishima, S. Konishi, and K. Honda, Nature (London) 277, 637 (1979).

[2] H. Kato and A. Kudo, Chem. Phys. Lett. 295, 487 (1998).

[3] K. Ikarashi, J. Sato, H. Kobayashi, N. Saito, H. Nishiyama, and Y. Inoue, J. Phys. Chem. B 106, 9046 (2002).

[4] J. Sato, N. Saito, H. Nishiyama, and Y. Inoue, J. Phys. Chem. B 105, 6061 (2001).

[5] J. Sato, H. Kobayashi, and Y. Inoue, J. Phys. Chem. B 107, 7970 (2003).

[6] J. Sato, H. Kobayashi, K. Ikarashi, N. Saito, H. Nishiyama, and Y. Inoue, J. Phys. Chem. B 108, 4369 (2004).

[7] X. Wang, K. Maeda, A. Thomas, K. Takanabe, G. Xin, J. M. Carlsson, K. Domen, and M. Antonietti, Nat. Mater. 8, 76 (2009).

[8] J. Liu, Y. Liu, N. Liu, Y. Han, X. Zhang, H. Huang, Y. Lifshitz, S.-T. Lee, J. Zhong, and Z. Kang, Science 347, 970 (2015).

[9] Q. G. Jiang, Z. M. Ao, D. W. Chu, and Q. Jiang, J. Phys. Chem. C 116, 19321 (2012).

[10] J. Wirth, R. Neumann, M. Antoniettib, and P. Saalfrank, Phys. Chem. Chem. Phys. 16, 15917 (2014).

[11] Y. Zheng, Y. Jiao, Y. Zhu, L. H. Li, Y. Han, Y. Chen, A. Du, M. Jaroniec, and S. Z. Qiao, Nat. Commun. 5, 3783 (2014).

[12] M. A. El-Sayed, Acc. Chem. Res. 34, 257 (2001).

[13] K. K. Kelly, E. Coronado, L. L. Zhao, and G. C. Schatz, J. Phys. Chem. B 107, 668 (2003).

[14] L. Brus, Acc. Chem. Res. 41, 1742 (2008).

[15] L. Yan, F. Wang, and S. Meng, ACS Nano 10, 5452 (2016).

[16] E. Runge and E. K. U. Gross, Phys. Rev. Lett. 52, 997 (1984).

[17] Y. S. Al-Hamdani, D. Alfè, O. Anatole von Lilienfeld, and A. Michaelides, J. Chem. Phys. 144, 154706 (2016).

[18] P. Ehrenfest, Z. Phys. 45, 455 (1927).
[19] Fundamental of Time-Dependent Density Functional Theory, edited by M. A. L. Marques, N. Maitra, F. Nogueira, E.K.U Gross, and A. Rubio, Lecture Notes in Physics, Vol. 837 (Springer-Verlag, Berlin, 2012).

[20] Y. Miyamoto, Y. Tateyama, N. Oyama, and T. Ohno, Sci. Rep. 5, 18220 (2015).

[21] A. Castro, M. A. L. Marques, J. A. Alonso, G. F. Bertsch, and A. Rubio, Eur. Phys. J. D 28, 211 (2004).

[22] J. P. Perdew and A. Zunger, Phys. Rev. B 23, 5048 (1981).

[23] J. P. Perdew, K. Burke, and M. Ernzerhof, Phys. Rev. Lett. 77, 3865 (1996).

[24] M. Suzuki, J. Phys. Soc. Jpn. 61, 3015 (1992).

[25] M. Suzuki and T. Yamauchi, J. Math. Phys. 34, 4892 (1993).

[26] J. Ihm, A. Zunger, and M. L. Cohen, Phys. C 12, 4409 (1979).

[27] N. Troullier and J. L. Martins, Phys. Rev. B 43, 1993 (1991).

[28] L. Kleinman and D. M. Bylander, Phys. Rev. Lett. 48, 1425 (1982)

[29] O. Sugino and Y. Miyamoto, Phys. Rev. B 59, 2579 (1999); 66 089901(E) (2002).

[30] Y. Miyamoto and H. Zhang, Phys. Rev. B 77, 165123 (2008).

[31] See Supplemental Material at http://link.aps.org/supplemental/ 10.1103/PhysRevB.96.115451 for [give brief description of material].

[32] H. Zhang and Y. Miyamoto, Appl. Phys. Lett. 95, 053109 (2009).

[33] H. Zhang, Y. Miyamoto, X. Cheng, and A. Rubio, Nano Scale 7, 19012 (2015).

[34] D. R. Hamann, Phys. Rev. B 55, R10157(R) (1997).

[35] M. S. C. Mazzoni, H. Chacham, P. Ordejón, D. Sánchez-Portal, J. M. Soler, and E. Artacho, Phys. Rev. B 60, R2208(R) (1999).

[36] M. F. Islam, E. Rojas, D. M. Bergey, A. T. Johnson, and A. G. Yodh, Nano Lett. 3, 269 (2003). 